\title{
Algorithm of Face Recognition Based on Chaotic theory
}

\author{
Yang Yu-ping \\ Computer College, Chongqing College of Electronic Engineering, Chongqing 401331, china \\ wwwyyp2010@163.com
}

Keywords: face recognition; chaotic projection; information security

\begin{abstract}
Face recognition belongs to the field of digital image, there are many applications. The classical algorithms of face recognition include algorithm of PCA, algorithm of LDA, algorithm of ICA, and algorithm of SIFT which is for robust feature extraction, etc. In this paper, the improved algorithm is proposed based on classical algorithm of SIFT, and the chaos theory is applied to the improved algorithm to project the datum of face images. It is proved that the improved algorithm has better anti-attack and better recognition efficiency.
\end{abstract}

\section{Introduction}

Face recognition technology which processes the input face image or video stream is based on human facial features [1]. First of all, the system judges whether or not a face is present. If there is a face, then the system would show us the location and size of each face, and location information of each major facial organ. And then extract the identity feature contained in each face and compare it with the known face to identify the identity of each face [2]. Face recognition technology consists of three parts: (1) face detection, the purpose is to separate the human face from the background image, generally there are (1) reference template method (2) face rules method (3) sample learning method (4) skin color model method (5) feature sub-face method, and the mixture method between them.; (2) Face tracking, face tracking is to track face of the dynamic target with detecting; (3) Face matching, Face matching is the identification of a face image that is detected or a target that is searched in a database of face images. The methods which are commonly used are: (1) eigenvector method (2) face template method [3].

\section{Chaos theory and face recognition process}

Chaos theory is a method of qualitative thinking and quantitative analysis, it is used to explore interpreted and predicted behavior with relationship of holistic, continuous rather than A single data in the dynamic system (such as: population movements, chemical reactions, weather changes, social behavior, etc.). in 1963, Edward Norton Lorenz who is a meteorologist in American proposed chaos theory, which is a diversity and multi-scale nonlinear system. Chaos theory explains that the decision system may produce random results [4]. The greatest contribution of chaos theory is the use of simple models to obtain explicit periodic results. There are some characteristics of chaotic theory: (1) Randomness: the system is chaotic because of irregular behavior, which is generated at random by internal dynamics in the system; (2) Sensitivity: the system's orbit is extreme sensitive on the initial value; (3) Fractal dimension: it refers to the geometry of system motion orbit in phase space, which can be described in fractal dimension;

(4) Universal: When the system tends to chaos, the characteristics of which is shown universal and significant; (5) Scaling: chaos phenomenon is a non-periodic ordered state, with an infinite level of self-similar structure in no scale region [5].

Chaos theory is very wide application. In different areas, according to the specific circumstances of the application, the abstract mathematical formulas are different, so currently there is no unified definition of the chaos [6]. There is a mathematical abstraction formula which is the use of more common is: 


$$
C H_{n+1}=r * C H_{n}-r * C H_{n}^{2}, \quad\{n \mid n \in[0,+\infty], n \in Z\}
$$

The value of $\mathrm{r}$ in is defined as $\{r \mid r \in(3.541,4)\}$. Formula (1) will generate a sequence of non-cyclical deterministic nonlinearities, that is, chaotic sequence.

Face recognition process generally in three steps [7]:

(1) First, the database establishment of facial images. The facial image data, which generate data files, is collected by the camera, and the files are coded and stored to storage, which is the registration database.

(2) To obtain the current face images. Captures the face image of the current person with the camera, and generates.

(3) Comparing the codes of current face image with the codes in the registration database of face images and searches.

Face recognition process can be completed by ordinary image processing equipment in real time, automatically and continuously [8].

\section{Application of chaotic projection in face recognition}

SIFT (Scale Invariant Feature Transform) algorithm is extraction algorithm based on local face feature. The SIFT algorithm is stable for extracting the local features of the human face, with the stable characteristics for rotation, scaling, brightness and so on. The following is the basic structure figure of SIFT algorithm of feature extraction in the face registration phase and identification phase, which is in the recognition system, as shown in figure 1 :

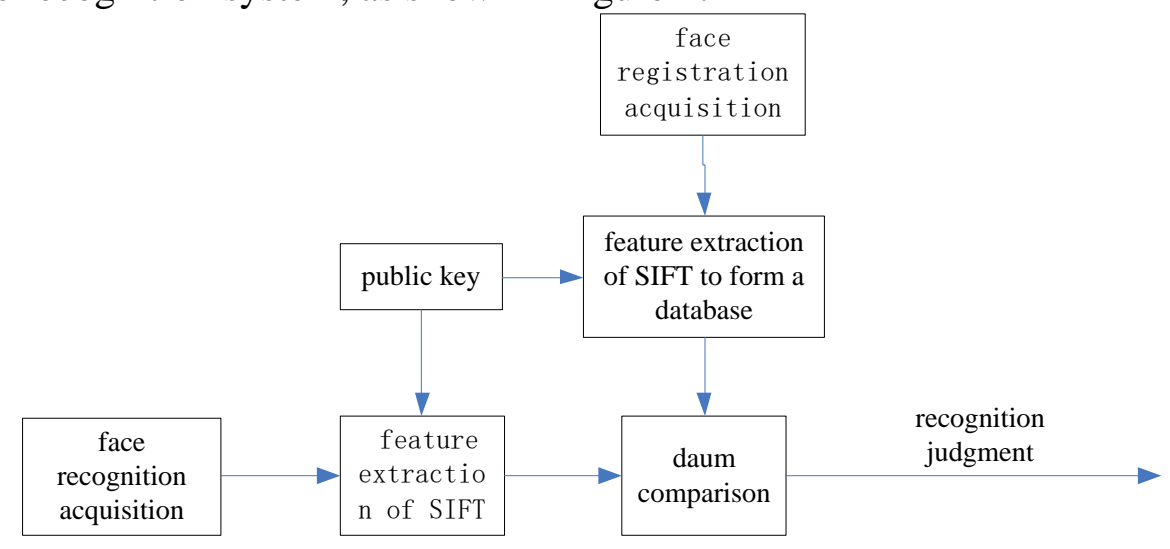

Figure 1 the basic structure of SIFT algorithm

From figure 1, it can be seen that the data in the face database is not the face images but the feature datum of the face images, but the feature datum are not encrypted in depth, with just only the public key, which is shared by many registered people, for feature datum encryption. That is to say, the administrator or developer of the recognition system can fully operate and use every person's characteristic datum in face feature database, which is a big security risk.

Therefore, we apply the chaos theory in this algorithm; the chaotic projection of the extracted feature datum enhances the security of the encryption of the feature datum, while the key for encryption is set by their own of registered person. The specific improvement of the algorithm is shown in Figure 2: 


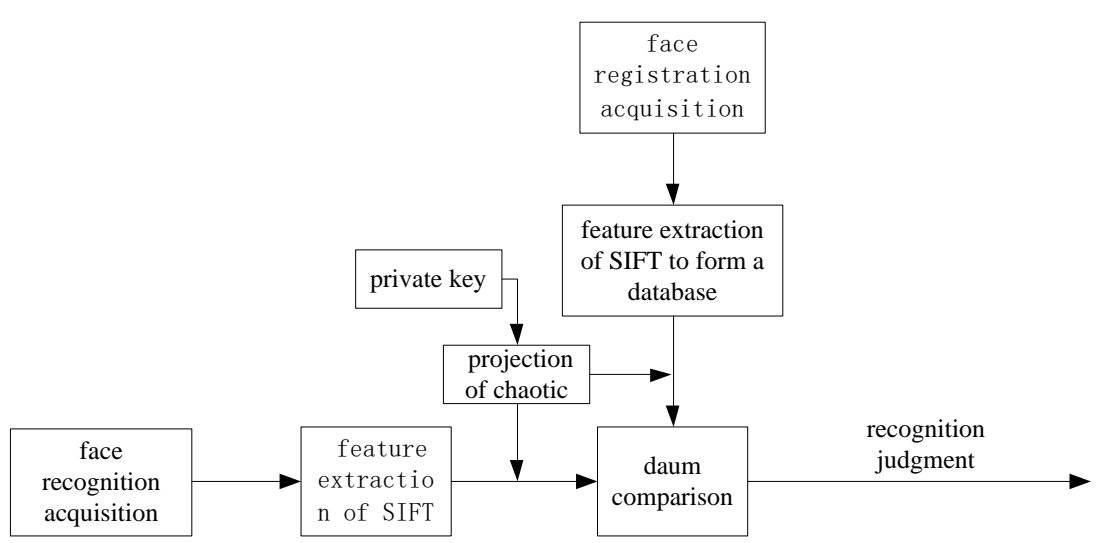

Figure 2 the structure of improve algorithm

As the general projection algorithm is relatively easy to be cracked, and not very stable. So we improve algorithm with the application of chaos theory, which makes the feature datum more security and stable after projection. Under normal circumstances, a recognition system with a shared key to encrypt is easy to be cracked, and the shared key would be easy to be tested out, when the registered staff is limited and the face images is easy to get by administer, and there is relatively large security risk.

The running steps of algorithm proposed used in the face recognition system are as follows:

1) Collect facial images of registration person, and enter their own personal key " $k$ " to build feature database of facial images of registration person.

2) Person waiting for identification put images of their faces by the camera of the face recognition system, and input their own personal key " $k$ ".

3) The registration system uses the input key " $k$ " as the initial value, and uses chaos formula (1) to generate a matrix R-matrix of chaotic projection.

4) The recognition system extracts the feature of face by the algorithm of SIFT feature extraction, and makes "and" operation with the matrix R-matrix of chaotic projection.

5) The registration system uses the result of "and" operation to compare with the datum in the database.

If the person to be identified is not legitimate user, who does not know the registered personal key "k", which can avoid security risk from management and system developers; if the registered person tell the key " $k$ " to others to cheat the system, but the system needs to capture the scene Images, so that eventually it lead to cheating unsuccessful. If you use the key test software to get the key " $\mathrm{k}$ ", because each person's facial datum are not the same and each person's " $k$ " is not the same, so it can't be frequently tempted for the small test datum, and it ultimately lead to test failure.

\section{Analysis of experimental datum}

1) This improved algorithm is mainly used in the AR face database. There are 120 people in AR face database; each person has 14 face images. We selected 7 people as registered face images, selected 7 people as the test face images, as shown in Figure 3.

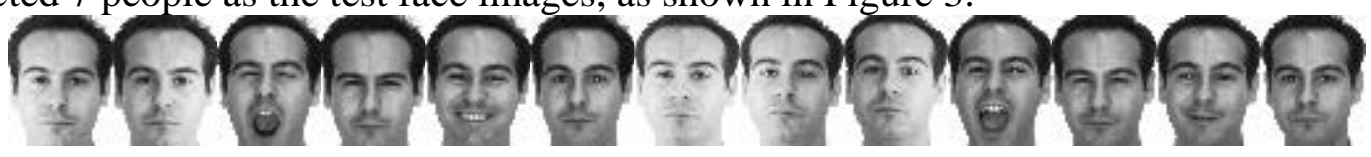

Figure 3 AR face database 
2) Analysis of the experimental effect

Table 1 improved algorithm based on SIFT compared with the algorithm of classic SIFT

\begin{tabular}{|c|c|c|c|c|c|c|c|c|c|}
\hline projection & imension & 120 & 110 & 100 & 90 & 80 & 70 & 60 & 50 \\
\hline \multirow{2}{*}{$\begin{array}{l}\text { SIFT } \\
\text { algorithm }\end{array}$} & $\begin{array}{l}\text { average recognition } \\
\text { rate }\end{array}$ & 0. 9939 & 0. 9926 & 0. 9924 & 0. 9935 & 0. 9918 & 0. 9933 & 0. 9920 & 0. 9919 \\
\hline & average time (s) & 0. 9400 & 0.8800 & 0. 8400 & 0. 7900 & 0. 7200 & 0. 6800 & 0. 6300 & 0. 5800 \\
\hline \multirow{2}{*}{$\begin{array}{l}\text { improved } \\
\text { algorithm }\end{array}$} & $\begin{array}{l}\text { average recognition } \\
\text { rate }\end{array}$ & 0. 9960 & 0. 9946 & 0. 9948 & 0. 9952 & 0. 9938 & 0. 9952 & 0. 9945 & 0. 9940 \\
\hline & average time (s) & 0. 9410 & 0. 8820 & 0. 8400 & 0. 7900 & 0. 6800 & 0. 6790 & 0. 6300 & 0. 5805 \\
\hline
\end{tabular}

In order to make the experimental datum more general, to reduce the errors caused by experimental conditions, we use the datum, which are taken from the average. As can be seen from Table 1, improved algorithm and classic SIFT algorithm almost take the same time while system running, but the recognition rate of improved algorithm increases. And also system carries out the identification of the illegal users (assume that the face images are misappropriated), and the realistic simulation of the recognition system is carried out. The authenticity of the improved recognition system is proved to be much better than that of the classic SIFT algorithm recognition system.

\section{Conclusion}

Face recognition is becoming more and more popular in people's life and work, and its safety has been paid more and more attention. Based on the classic SIFT algorithm, this paper applies the chaotic theory for the projection of the facial feature datum to encrypt, and uses the personal key of the registered person, which makes the system more secure.

\section{References}

[1]Li fang Wu, LAN sun Sheen. Face recognition from front-view face [J].Journal of Electronics (China), 2003, 201:

[2]Ping Shan Liu, Han ping Lu, Song de Ma. A non-parameter Bayesian classifier for face recognition [J].JournalofElectronics, 2003, 205:

[3]Wee Zhen Xian, Wee-wee Zhang. The influence of emotion while face processing [J]. Cognition and Emotion, 2016, 302:

[4] YvonGauthier. The Construction of Chaos Theory [J].FoundationsofScience, 2009, 143:

[5]Warren Smith, Matthew Higgins. Postmodernism and Popularization: The Cultural Life of Chaos Theory [J].CultureandOrganization, 2003, 92:

[6]Salvo Chetek. Applying chaos theory to lesson planning and delivery [J].European Journal of Teacher Education, 2008, 313:

[7]ManuelaMalaspina, ReaAlbonico, RobertaDaini. Right perceptual bias and self-face recognition in individuals with congenital prosopagnosia [J]. Laterality: Asymmetries of Body, Brain and Cognition, 2016, 212:

[8]A.J.Dowsett, A.Sandford, A.MikeBurton. Face learning with multiple images leads to fast acquisition off familiarity for specific individuals [J].The Quarterly Journal of Experimental Psychology, 2016,691: 